\title{
Policentrismo en el Área Metropolitana de Santiago de Chile: reestructuración comercial, movilidad y tipificación de subcentros ${ }^{1}$
}

Ricardo Truffello. Universidad Adolfo Ibáñez, Santiago, Chile.

Rodrigo Hidalgo. Pontificia Universidad Católica de Chile, Santiago, Chile.

RESUMEN | El complejo proceso de evolución de las áreas metropolitanas ha reconfigurado las estructuras urbanas, pasando desde los modelos monocéntricos a nuevos patrones basados en el paradigma del policentrismo. Se aborda esta problemática, para el Área Metropolitana de Santiago, por medio del análisis de datos de densidad comercial y precios de suelo, utilizando la aplicación de modelos paramétricos. Los resultados indican una evolución hacia una estructura urbana policéntrica comercial que aún coexiste con algunos rasgos de monocentrismo. Se identificaron diecinueve candidatos a subcentro comercial, los que fueron evaluados, clasificados y caracterizados mediante la utilización de variables complementarias a la densidad de superficie de uso comercial, como la movilidad. Adicionalmente se confirmó la validez del uso de la variable superficie comercial y se encontró en el modelo exponencial negativo la mejor fórmula de ajuste paramétrico para la ciudad de Santiago.

PALABRAS CLAVE | estructura urbana, metropolización, reestructuración territorial.

ABSTRACT | The complexity in the evolution of metropolitan areas has transformed urban structures from classical monocentric models to new models based on the polycentric paradigm. This phenomenon has been approached in the case of the Metropolitan Area of Santiago, through the analysis of commercial density and land price data, applying parametric modeling in the analysis. The results indicate an evolution towards a polycentric urban structure that coexists with some remaining traits of the monocentric structure. Nineteen candidates for commercial sub-centers were identified, evaluated and classified through the use of variables complementary to commercial land use density, such as mobility indicators. In addition, the use of the commercial land use variable was validated, and the negative exponential function model was found to be the best formula for parametric adjustment in the case of the Metropolitan Area of Santiago.

KEYWORDS | urban structure, metropolization, territorial restructuring.

Recibido el 8 de marzo de 2012, aprobado el 25 de abril de 2013

E-mail: ricardo.truffello@uai.cl | hidalgo@geo.puc.cl

Correspondencia autor principal: Universidad Adolfo Ibańez, Centro de Inteligencia Territorial, Presidente Errázuriz 3485, Las Condes, Santiago, Chile.

1 Este artículo es producto del proyecto Fondecyt 1095122, titulado "La transformación de las áreas centrales: reestructuración comercial y elitización (gentrificación) residencial. El caso de Santiago, Valparaíso y Vińa del Mar". Se agradece el Proyecto conicyt/fondap/15110020 (Cedeus) y al Proyecto CONICYT/FONDAP/15130009 (COES). 


\section{Introducción}

El presente artículo discutirá la evolución de las áreas urbanas en el contexto de la metropolización y el cambio de paradigma desde modelos monocéntricos a otros policéntricos; posteriormente se analizarán los factores, variables y métodos registrados en la literatura para delimitar, identificar y caracterizar subcentros. En este contexto se define como objetivo del estudio la identificación de candidatos a subcentralidades comerciales, para lo cual se realizan modelaciones monocéntricas con dos variables dependientes (precios de suelo y densidad de uso comercial), tres variables independientes (distancia, movilidad pública y privada, todas medidas con análisis de redes), seis modelos de regresiones y dos momentos temporales (20012009). A partir de los resultados se escogen dos modelos monocéntricos, se presentan y analizan los candidatos a subcentros y se procede a realizar una modelación aditiva (policéntrica). A partir de esta, se examina la estructura urbana de Santiago y su evolución temporal. Finalmente se realiza una caracterización cualitativa de los candidatos a subcentros.

\section{Evolución de las áreas urbanas y paradigma del policentrismo}

Desde mediados del siglo pasado, el proceso de evolución de las áreas urbanas, y particularmente de las metrópolis, ha cambiado radicalmente. Esto encuentra explicación en factores como el acelerado crecimiento de la población, los procesos de suburbanización, la inmigración, las diferencias en la tipología de los hogares, la desconcentración de las actividades económicas y el uso de las tecnologías de la información (Kloosterman \& Musterd, 2001).

La compleja interacción de los factores mencionados ha derivado en fenómenos espaciales de dispersión urbana y concentración descentralizada (Dematteis, 1996), lo que se manifiesta principalmente en las grandes áreas metropolitanas por medio de conjuntos de centros de diferentes tamańos y jerarquía que se interrelacionan en distintos ámbitos (Rojas Quezada, Muñiz Olivera \& García-López, 2009), como el espacial, administrativo, funcional y cultural (Kloosterman \& Musterd, 2001). En el marco de estos patrones, la ciudad -específicamente las áreas metropolitanasadquiere una configuración espacial que se aleja de las propuestas por las teorizaciones clásicas, nacidas a partir del modelo de Von Thünen (Ruiz \& Marmolejo Duarte, 2008), lo que hace indispensable centrar el análisis cuantitativo en nuevos modelos que asuman la existencia de centros múltiples o subcentros; es decir, modelos que funcionen bajo el paradigma del policentrismo urbano.

Las líneas de investigación "policéntricas”, lideradas desde la Geografía Económica, han desarrollado estudios con diversas perspectivas: en un caso, se analiza el policentrismo a partir de diferentes modelos teóricos, como el de la Renta Ofertada o el del Lugar Central (Muñiz, Galindo \& García, 2005); en otro, se enfoca el estadio policéntrico desde la propia escala del fenómeno, regional e intraurbana (Parr, 2004); y un tercer enfoque toma en consideración la modelación con diferentes 
variables y métodos, como la densidad, los precios de suelo (modelos paramétricos), y funcionales y de movilidad (no paramétrico) (Roca, Marmolejo \& Moix, 2008).

A pesar de la diversidad de enfoques y métodos en los estudios y líneas de investigación, los trabajos de policentralidad han apuntado a explicar este fenómeno y la respectiva transición de los modelos monocéntricos hacia la policentralidad, a partir de la relocalización del empleo (Muñiz, Galindo \& García, 2005), siendo las investigaciones de policentralidad sobre la base del empleo comercial mucho más escasas (Ruiz \& Marmolejo Duarte, 2008).

En el caso de Chile, la constante se repite con una tradición aún menor de precedentes. Como referentes se puede tomar el estudio realizado en el Área Metropolitana del Gran Concepción (Rojas Quezada, Muñiz Olivera \& García-López, 2009), el que se basa en los preceptos funcionales derivados de la relocalización del empleo (con métodos de análisis de densidad). Se suma al anterior un trabajo sobre el Área Metropolitana de Santiago (Ortiz \& Escolano, 2005), que aborda la policentralidad de la referida ciudad desde la perspectiva del empleo comercial, utilizando medidas de densidad no paramétricas y de movilidad.

Es necesario explorar las diferentes aristas metodológicas que permitan definir la estructura urbana de la ciudad en función de sus características de centralidad, considerando que dicha modelación servirá como aporte a la línea de investigación y definición de estructuras urbanas y policentralidad, para el caso chileno y en especial para el Área Metropolitana de Santiago (AMS). En tal sentido, parece relevante poner en cuestión la validez del modelo solo para el área analizada (independientemente del carácter metodológico de la investigación y su resultado), ya que las características de la policentralidad -y, por tanto, de la estructura urbana de un área metropolitana- dependen no solo de las variables analizadas, sino también de su historia y evolución particular - principal crítica de Krugman y Fujita (1997) a los modelos de la Escuela Alemana-.

En función de todas estas características se conforma un interesante escenario para la elaboración de una investigación ligada al análisis de la estructura urbana comercial del Ams. Esto con el objetivo de responder a interrogantes como: ¡cuáles son las variables más adecuadas para definir centralidad comercial en el AMs?; ¿̨cuáles son los métodos más efectivos para definir candidatos a subcentros en el contexto del análisis paramétrico de la centralidad comercial?; ¿cómo han evolucionado las centralidades comerciales en el AMs?; los malls ¿son candidatos reales a subcentros comerciales?; ¿es posible definir policentralidad a partir de la aglomeración comercial? Y si es así, ¿es el AMs un sistema urbano policéntrico?

\section{¿Centralidad o centralidades? Desde el modelo monocéntrico al policéntrico}

En el contexto urbano, las economías de aglomeración generan aumentos productivos derivados de la concentración de la oferta de bienes y servicios, y a la vez de personas que acuden a ellos a satisfacer sus necesidades de consumo (Polèse, 2001). El agrupamiento de la cantidad, calidad, variedad y densidad de elementos ofertados favorece, como elemento básico, la formación de una centralidad, que por excelencia ha estado aglutinada en lo que la tradición anglosajona ha definido 
como el Distrito Central de Negocios, el CBD (Central Business District) (Horwood \& Boyce, 1959).

Esta concentración, una vez llegada a un punto crítico, no solo genera beneficios, sino también importantes deseconomías que, de acuerdo con lo expresado por Krugman (1999), actúan como fuerzas centrífugas. Estos procesos van acompañados del crecimiento demográfico y espacial de las ciudades, cuestión que complejiza el análisis de las estructuras urbanas en las que se materializan. Esto redunda en nuevas áreas metropolitanas que, desde la perspectiva de su estructura urbana, se alejan de los modelos monocéntricos, dando lugar en un principio a nuevas subcentralidades, para terminar finalmente en un policentrismo formal.

El policentrismo se puede definir como un proceso mediante el cual la estructura de la ciudad se aleja de forma progresiva de una estructura monocéntrica (caracterizada principalmente por la agrupación de funciones comerciales y de servicios en un solo centro), a una donde coexisten varios centros de empleo, que ofertan bienes, servicios y diversas formas de satisfacción de necesidades para la población que concurre a ella con diversos intereses (Muñiz, Galindo \& García, 2005).

En síntesis, el policentrismo, bajo sus diferentes formas, se construye con la aparición de subcentros, los que, por su dinámica, abastecen parcial o totalmente la demanda de zonas de la ciudad que por distancias o tiempos de viaje tienen menores índices de conectividad y accesibilidad respecto del CBD. Conceptualmente, el subcentro corresponde así a una unidad territorial articuladora en la ciudad, que posee una alta densidad de empleos y de oferta de bienes y servicios y que, en consecuencia, genera altos flujos de viajes, vale decir, alta movilidad (Ruiz \& Marmolejo Duarte, 2008).

Por la misma lógica de la policentralidad, los subcentros asumen múltiples patrones dentro de la ciudad. De hecho, corresponden a modelos diferentes, según sean los factores intrínsecos vinculados a su creación y, posteriormente, a su funcionamiento. No obstante, en términos generales es posible clasificarlos -según sus usos predominantes- como residenciales, o bien comerciales y de servicios. En ambos casos, el factor común que los definirá como subcentros es la alta densidad sea comercial, de servicios o residencial (Ruiz \& Marmolejo Duarte, 2008). Para el caso particular de esta investigación, la delimitación y tipificación de los mismos se desarrollará considerando su arista comercial.

\section{Delimitación y tipificación de subcentros comerciales: factores, variables y métodos}

\section{Factores}

Parte importante de los objetivos de la investigación se centra en establecer una comparación territorial de los resultados paramétricos (subcentralidades comerciales y su tipificación) arrojados por los diferentes análisis abordados. Para esto resulta fundamental contar con una unidad territorial que sea capaz de recibir los datos correspondientes a cada uno de los métodos. Tomando como referencia los trabajos desarrollados en España, y particularmente en Barcelona (Roca, Marmolejo \& Moix, 2008; Marmolejo, 2013; y Ruiz \& Marmolejo Duarte, 2008), parece necesario considerar la unidad político-administrativa correspondiente a los municipios, ya 
que constituye una unidad territorial que tiene injerencia sobre la ciudad, facilita la aplicación del método de agregación espacial, pero además permite entregar los resultados en un formato conocido por especialistas en la materia que aborda el artículo. No obstante tales ventajas, para el AMs ese enfoque resulta inoperable, debido a dos factores: el tamaño de las comunas y, derivado de lo mismo, la heterogeneidad de cada una de ellas ${ }^{2}$. Esta heterogeneidad no es solo espacial, sino que también social, lo que se traduce en una segregación que se ha reescaldo, dejando de lado la separación entre las grandes zonas de la ciudad (ganadoras y perdedoras, ricas y pobres), para dar paso a una microsegregación (Borsdorf, Hidalgo \& Sánchez, 2007; Hidalago \& Borsforf, 2009). Esta microsegregación, para efectos del estudio, se convierte en un problema que dificulta la calificación de las áreas analizadas bajo un solo y único criterio.

Para alojar los resultados del análisis paramétrico llevado a cabo en este estudio, se delimitaron zonas homogéneas considerando factores de usos de suelo, grupos socioeconómicos, tipologías de construcción y límites de barrio (Figura 1). Ello permitió establecer 794 zonas a las que se les asignó un CBD con medidas de densidad de superficie de uso comercial de corte no paramétrico (análisis de peaks ${ }^{3}$ ).

\section{Variables}

La densidad de empleos -en particular la densidad comercial- aparece como la variable más utilizada para la identificación de subcentros. De hecho, es aquella que, desde la perspectiva empírica, ha sido abordada de forma más profusa por metodologías y métodos de análisis econométricos, permitiendo la generación de modelos cada vez más precisos y consistentes (Marmolejo, 2013). No obstante no es la única.

La distancia entre subcentros y las distancias de estos con el СвD aparece como un factor muy relevante, según Roca et al. (2008), ello se explica por la interrelación sistémica que se da en una estructura urbana policéntrica. Pero estos autores van más allá, especificando que el análisis de la movilidad es el que permite detectar los verdaderos subcentros, entendidos como unidades articuladoras de la estructura urbana donde confluyen los flujos más significativos que se generan en las aglomeraciones urbanas.

Entre las variables que destacan por su utilización en los estudios de esta línea, se pueden mencionar también los viajes realizados dentro de una ciudad. En esta perspectiva se los entiende como movilidad y se los define como densidad de viajes; más específicamente, como el espesor de viajes por motivos comerciales (Gordon \& Richardson, 1996). También destaca la utilización del precio de suelo, entendiendo que esta variable se convierte en una medida resumen de factores como los usos permitidos, la distancia temporal al centro histórico, los flujos de personas

2 En el Área Metropolitana de Santiago las comunas son, por su tamaño (6.523 ha en promedio), altamente heterogéneas, y ello en varias dimensiones; por ejemplo, heterogeneidad socioeconómica, como en el caso de Peñalolén, que presenta vivienda social y condominios de altos ingresos mezclados en el territorio; o de uso de suelo, como en Providencia, en cuyo territorio hay áreas netamente residenciales, áreas de uso de oficina y extensas áreas comerciales.

3 Análisis de densidad espacial que definió el CBD por medio de la determinación de los máximos obtenidos en función de los datos de densidad de uso comercial y precios de suelo. 
del sector y la calidad de los servicios disponibles (Núñez \& Schovelin, 2002). De hecho, su aplicación se ha utilizado para modelar, predecir y entender la estructura de la ciudad (McMillen, 1996).

FIGURA 1 | Proceso para la creación de zonas homogéneas y zonas vs. comunas

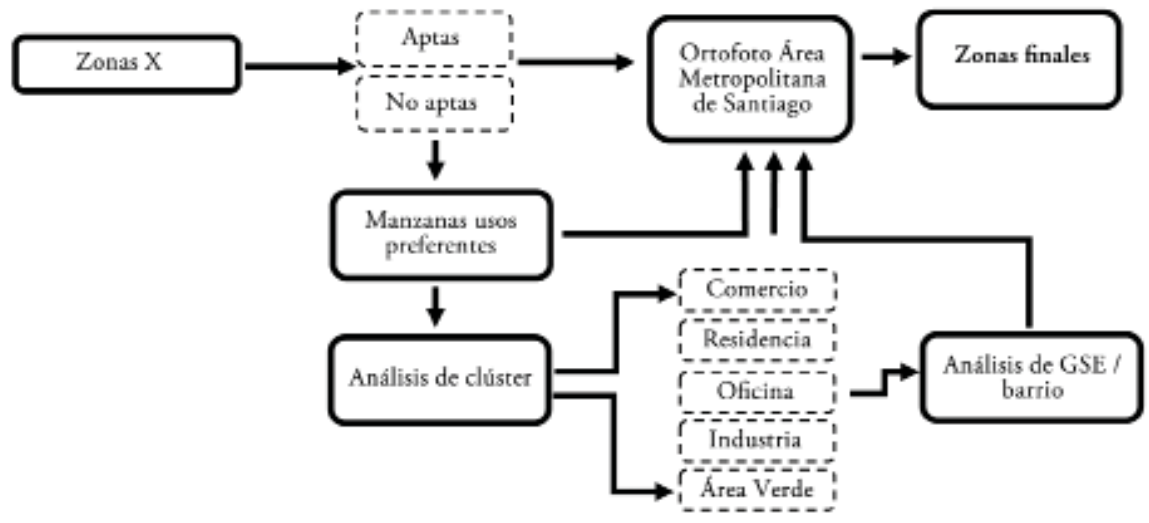

Zonas homogéneas AMS

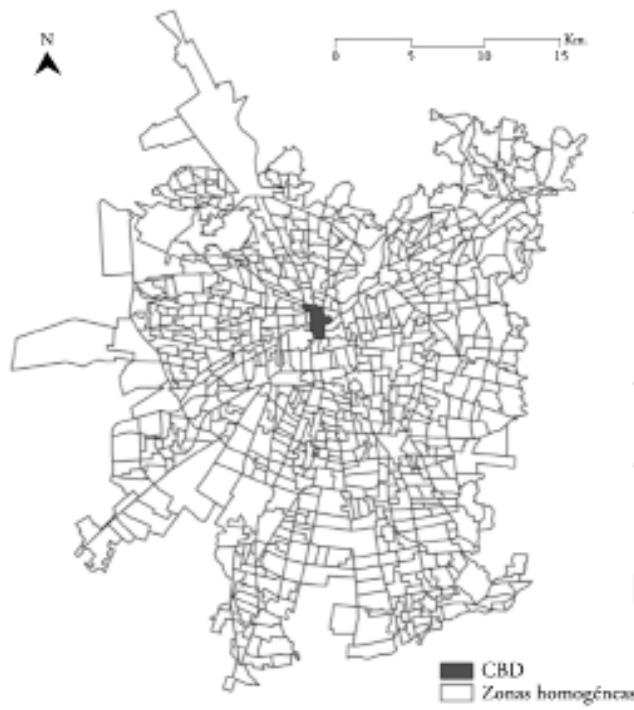

Comunas AMS

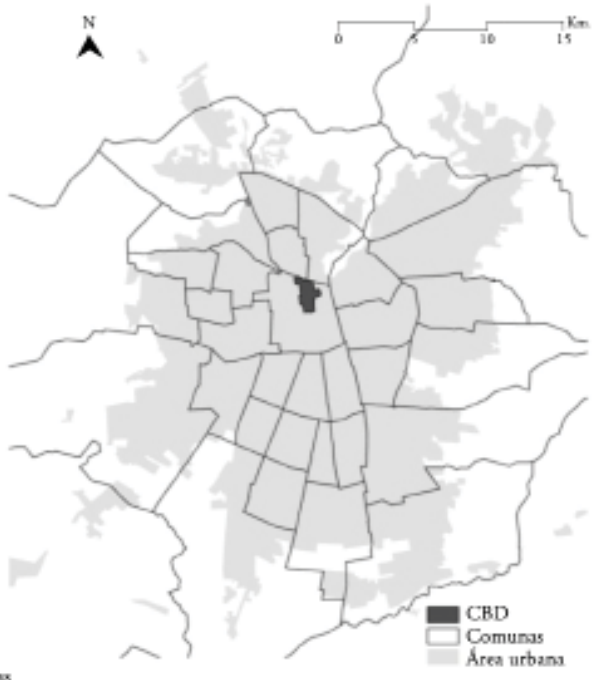

FUENTE ELABORACIÓN PROPIA

En función de dichos preceptos teóricos y considerando que no existe en Chile un catastro totalizante y georreferenciado de empleos, se utilizaron tanto variables de precio de suelo ${ }^{4}$ en superficie de uso comercial por manzana ${ }^{5}$ para la ciudad de Santiago, como variables dependientes.

4 Datos de oferta extraídos del Portal Inmobiliario (www.portalinmobiliario.com/), para los ańos 2009 y 2011.

5 Superficie de uso comercial catastrada por el Servicio de Impuestos Internos, para los años 2001 y 2009. Generalizada como densidad a nivel de zonas. 
FIGURA 2 | Variables independientes utilizadas (distancia y movilidad)

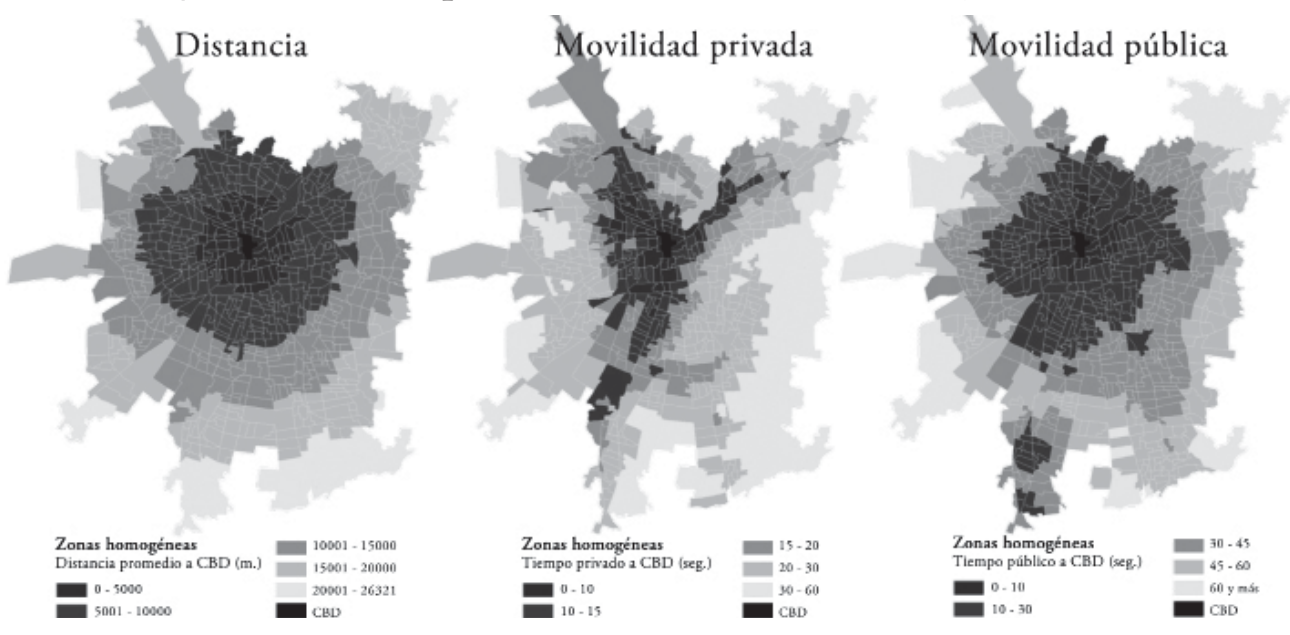

FUENTE ELABORACIÓN PROPIA

Como variable independiente se ocupó la distancia, movilidad privada y pública al $\mathrm{CBD}^{6}$ (desde las zonas homogéneas y calculadas con análisis de redes ${ }^{7}$ ), como forma para comparar la mejor alternativa para la modelación paramétrica (Figura 2).

\section{Métodos}

Con las variables clave definidas para la determinación de las posibles subcentralidades comerciales, se realizó un barrido de las metodologías empleadas para la detección e identificación de subcentros, tomadas a partir de los textos de Roca y sistematizados por Ruiz (véase Roca et al., 2008; y Ruiz \& Marmolejo Duarte, 2008).

\section{FIGURA 3 Evolución temporal para metodología de análisis de subcentralidades comerciales}

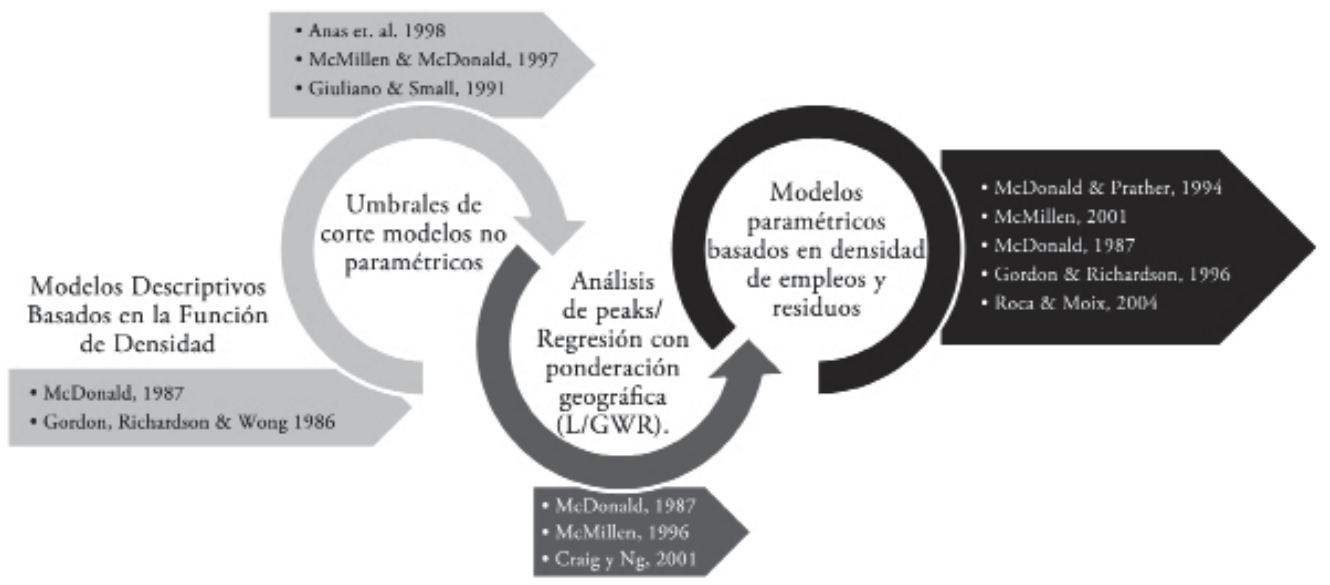

FUENTE ELABORACIÓN PROPIA SOBRE LA BASE DE RUIZ Y MARMOLEJO DUARTE (2OO8); Y MUÑIZ, GALINDO Y GARCÍA (2003)

6 Definido como el centro histórico de Santiago, en función de los máximos (peaks) de ambas variables.

7 Movilidad real calibrada por medio de los ejes viales de Santiago, modeladas con el módulo Network Analyst de ArcGIS. 
El cálculo de subcentros comerciales fue evaluado en función de la densidad de superficie comercial y precios de suelo, considerando metodologías paramétricas de análisis de residuos con diferentes modelos de regresión. Los modelos testeados en la investigación se indican en el Cuadro 1.

CUADRo 1 | Modelos de regresión paramétricos

\begin{tabular}{|c|c|}
\hline MODELOS APLICADOS & FORMULACIÓN \\
\hline Lineal (L) & $D(x)=b x+k$ \\
\hline Exponencial negativo (EXP) & $D(x)=a e^{b x+k}$ \\
\hline Exponencial negativo - versión logarítmica (estándar) & $\operatorname{Ln} D(x)=-b x+k$ \\
\hline Potencial inverso, o gravitatorio (GRAV) & $D(x)=a x^{b+k}$ \\
\hline Gravitatorio - versión logarítmica (log-log) & $\operatorname{Ln} D(x)=b \operatorname{Ln}(x)+k$ \\
\hline Potencial. & $D(x)=[(a \cdot x+k) \cdot(1-b)]^{(1 /(1-b))}$ \\
\hline \multicolumn{2}{|c|}{$\begin{array}{l}\text { Donde: } D(x) \text { es densidad comercial o precio de suelo; } \operatorname{Ln} D(x) \text { es logaritmo natural de densidad co- } \\
\text { mercial o precio de suelo; } x \text { es distancia o tiempo público o privado al CBD; } \operatorname{Ln}(x) \text { es logaritmo natural } \\
\text { de distancia o tiempo público o privado al CBD; } \boldsymbol{a} b \boldsymbol{k} \text { son constantes de ajustes, parámetros de las } \\
\text { diferentes regresiones presentadas. }\end{array}$} \\
\hline
\end{tabular}

FUENTE ELABORACIÓN PROPIA SOBRE LA BASE DE RUIZ \& MARMOLEJO DUARTE ( 2008).

La importancia de aplicar estos seis modelos de regresión tiene que ver con el ajuste de los mismos en función de la especificidad geográfica y espacial de cada ciudad. Dependiendo de la cantidad de candidatos a subcentros comerciales, las distancias al СвD y la distribución espacial de los mismos se pueden obtener diferencias significativas en los ajustes paramétricos. Por ende, no existe un modelo mejor que otro, sino que cada ciudad y su distribución espacial deben evaluarse de forma particular e independiente.

\section{Estimación de modelos paramétricos}

Para la aplicación de los modelos paramétricos antes presentados, es necesario realizar una estimación de los mismos, situación que incluye la elección de: la variable correcta, el modelo adecuado y los candidatos a subcentros (Figura 4).

Los insumos utilizados son los precios de suelo ${ }^{8}$ para los años 2009 y 2011, y a la densidad de uso comercial ${ }^{9}$ para 2001 y 2009. Estas variables dependientes fueron testeadas con las seis ecuaciones de regresión, incluyendo tres iteraciones por variable independiente (distancia, tiempo público y tiempo privado). El resumen de los resultados se presenta en el anexo del artículo (debido a la extensa cantidad de tablas derivadas de las combinaciones entre variables dependientes, independientes y modelos de regresión).

Portal Inmobiliario, precios de oferta.

9 Servicio de Impuestos Internos, superficie de uso comercial. 
FIGURA 4 Flujo metodológico análisis paramétrico de residuos

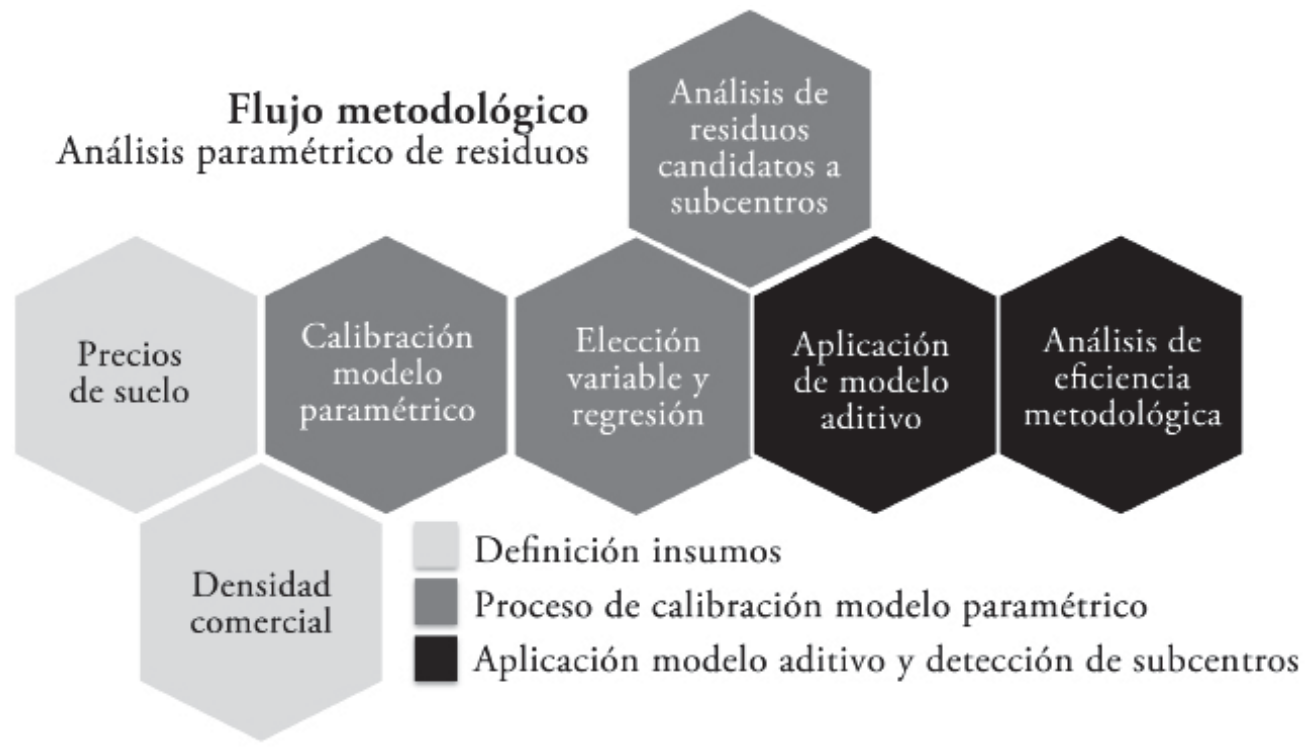

FUENTE ELABORACIÓN PROPIA SOBRE LA BASE DE RUIZ \& MARMOLEJO DUARTE (2008)

Los mejores resultados en función de los coeficientes de determinación y errores estimados fueron los siguientes:

CUADRO 2 | Mejores resultados etapa de calibración

\begin{tabular}{|l|c|c|}
\hline COLUMNA I & $\begin{array}{c}\text { EXP. DENSIDAD COMERCIAL 200I / } \\
\text { DISTANCIA }\end{array}$ & $\begin{array}{c}\text { EXP. DENSIDAD COMERCIAL 2009 / } \\
\text { DISTANCIA }\end{array}$ \\
\hline R2 & 0,483 & 0,391 \\
\hline K & 5,739 & 5,953 \\
\hline a & 14,290 & 11,711 \\
\hline b & $-0,461$ & $-0,447$ \\
\hline Error est. & 372,91 & 455,62 \\
\hline F. & - & - \\
\hline Sig & - & - \\
\hline
\end{tabular}

FUENTE ELABORACión PROPIA SObRe LA BASE DE RUIZ \& MARMOLEJO DUARTE (2008)

Como se observa en el Cuadro 2, los mejores resultados fueron obtenidos por el modelo exponencial negativo, para la variable dependiente de densidad comercial y variable independiente de distancia. El modelo dio por resultado 21 candidatos a subcentros ${ }^{10}$ (véase sección "Delimitación y tipificación de subcentros comerciales"), los que deben ser ingresados a la fórmula aditiva con el objeto de verificar la consis-

10 Cabe destacar que para la detección de los candidatos a subcentros comerciales se utilizó como corte dos desviaciones estándar, apelando a la menor calidad de los datos y la ya mencionada utilización de una variable proxy (densidad comercial) frente al escenario ideal (densidad de empleos comerciales). 
tencia del modelo y modelar el policentrismo. Para terminar, dichos subcentros se clasificaron por medio de un análisis mixto (cuantitativo y cualitativo) en función de las características de los mismos y variables de movilidad.

\section{Modelación policéntrica. Formulación aditiva (análisis de eficiencia metodológica)}

El cálculo de la formulación aditiva permite incorporar una nueva distancia a los subcentros detectados, estableciendo una modelación de la estructura urbana de Santiago bajo una hipótesis de comportamiento policéntrico.

El modelo en su fórmula aditiva -fórmula exponencial negativa en su variante aditiva $^{11}$ (Ruiz \& Marmolejo Duarte, 2008)- es:

$$
D(x)=a 1 e^{-b 1 \cdot D i s t C B D}+a 2 e^{-b 2 \cdot D i s t S u b C}+k
$$

Por parte de la variable independiente, la obtención de las distancias mínimas a subcentros (SBC) у СBD requiere la creación de una matriz de origen-destino, basada -para este caso- en la distancia. En el Cuadro 3 se presentan los resultados de la calibración con la fórmula aditiva.

CUADro 3 | Modelos densidad de superficie comercial en función de distancia a CBD y SBC

\begin{tabular}{|l|c|c|}
\cline { 2 - 3 } \multicolumn{1}{c|}{} & EXPONENCIAL 200I & EXPONENCIAL 2009 \\
\hline R2 & 0,764 & 0,772 \\
\hline K & 60,718 & 50,373 \\
\hline a1 & $3.322,05$ & $3.377,63$ \\
\hline b1 & 4,766 & 4,493 \\
\hline a2 & $2.038,74$ & $2.276,52$ \\
\hline b2 & 4,847 & 28,9 \\
\hline Error est. & 252,05 & 279,06 \\
\hline F. & - & - \\
\hline Sig & - & - \\
\hline
\end{tabular}

FUENTE ELABORACIÓN PROPIA SOBRE LA BASE DE RUIZ \& MARMOLEJO DUARTE (2008)

En el Cuadro 3 se puede constatar el aumento del ajuste y de la eficiencia del modelo exponencial. Este eleva significativamente su coeficiente de determinación (exponencial R2 = 0,764/0,772), siguiendo una tendencia común. Lo anterior da pistas para inferir una evolución hacia una estructura tendientes al policentrismo. A pesar de esto, cabe destacar la presencia de heterocedasticidad entre los datos, situación que debe redundar en una corrección de la misma a fin de validar los resultados de forma definitiva.

11 Para ver el significado de los elementos de la ecuación, remitirse a Cuadro 1. 
En 2001 ya se obtienen evidencias de policentrismo comercial. Si bien el CBD da cuenta de casi el 50\% del modelo monocéntrico (Gráfico 1), el hecho de alcanzar más de un $70 \%$ de varianza explicada para el modelo policéntrico (fórmula aditiva) permitiría inferir la existencia de áreas de mercado en Santiago que se rigen en función de centralidades que no corresponden al centro histórico de la ciudad. Esto ya era previsto por autores como Ortiz y Escolano en el año 2003 (Ortiz \& Escolano, 2005); sin embargo, la verificación paramétrica permite confirmar esta hipótesis de forma cuantitativa.

\section{GRÁfICO 1 | Coeficiente de determinación modelo exponencial}

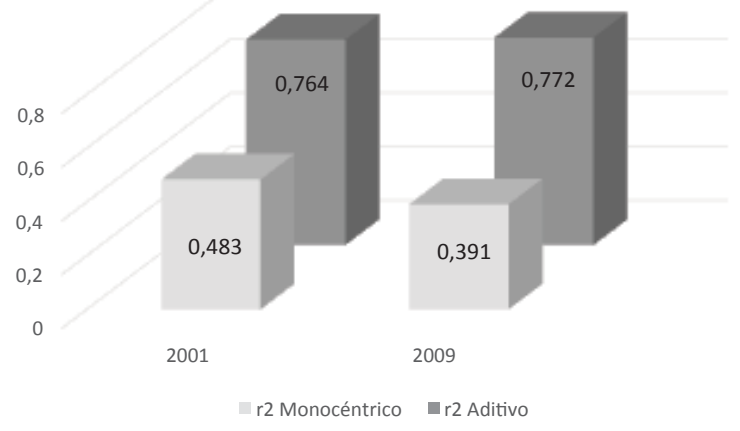

FUENTE ELABORACIÓN PROPIA

Según la serie de datos considerada, con los resultados para el año 2009 (véase Cuadro 3 y Gráfico 1), la hipótesis se confirma por dos hechos: i) el CBD pierde relevancia en la explicación del modelo monocéntrico, lo que en principio da luces de la disminución de la importancia del centro histórico para el Gran Santiago; y ii) ello da paso a la aparición de nuevas centralidades comerciales. Estas, en su conjunto, pesan casi tanto como el $\mathrm{CBD}$, lo que confirma una tendencia hacia una policentralidad comercial cada vez más acentuada.

La evolución de la ciudad hacia un modelo policéntrico formal dependerá a su vez de dos factores. El primero de ellos, la consolidación de los subcentros comerciales mediante el crecimiento de la oferta de servicios complementarios al ámbito comercial (situación que se da en zonas como el paradero 14 de la avenida Vicuña Mackenna, la avenida Providencia y calles aledañas, y algunos malls del AMs) (véase Figura 5). El segundo y más importante, las tendencias de crecimiento del AMs, caracterizado por la fragmentación de la periferia y con distintos componentes socioeconómicos de movilidad (accesibilidad, conectividad) así como densidad residencial de ocupación del suelo (diferencias entre clases sociales y tamaño de las viviendas).

En síntesis, se puede concluir que al año 2009 -en comparación con el 2001- el AMs tiene una estructura policéntrica comercial, debido a la aparición, consolidación y crecimiento de nuevos subcentros comerciales en la ciudad, los que ejercen influencia sobre la ciudad, en áreas hipotéticas de mercado de menor tamaño que el CBD. No obstante, no se debe dejar de lado la importancia marcada que tiene el centro histórico 
de Santiago, situación que no solo está remitida a la perspectiva comercial, sino a su condición de centro de empleos, equipamiento y servicios.

\section{Caracterización de subcentros comerciales}

A partir de la estimación e identificación de los subcentros comerciales, se procedió a profundizar en su categorización, para lo cual se establecieron algunos cruces de información que permitieran esclarecer esta interrogante. Puntualmente, se analizaron las características del subcentro en función de la modelación y su cruce con la información de movilidad, derivada de la Encuesta Origen Destino de 2006 (en adelante EOD) aplicada por la Secretaría de Planificación de Transporte (seCtra). También se exploró la distribución de los subcentros en el territorio de Santiago como respuesta a la utilización de otras variables, que puedan además justificar el porqué de su clasificación.

CUADRO 4 | Candidatos a subcentro. Resumen de información para identificación y medición

\begin{tabular}{|c|c|c|c|c|c|c|c|}
\hline NOMBRE & $\begin{array}{c}\text { DIST. } \\
\text { CBD } \\
(\mathrm{KM})\end{array}$ & $\begin{array}{c}\text { SUP. } \\
\text { COMERCIAL } \\
\text { (M2) }\end{array}$ & $\begin{array}{c}\text { VIAJES } \\
\text { TOTALES } \\
\text { (SEMANA) }\end{array}$ & $\begin{array}{c}\text { VIAJES } \\
\text { COMERC. } \\
\text { (SEMANA) }\end{array}$ & $\begin{array}{c}\% \text { VIAJES } \\
\text { TOTALES }\end{array}$ & $\begin{array}{l}\text { \% VIAJES } \\
\text { COMERC. }\end{array}$ & $\begin{array}{l}\text { RADIO }\left(^{*}\right) \\
\text { COM./TOT. }\end{array}$ \\
\hline CBD & 0 & 5.902 & 1.209 .468 & 184.661 & $2,67 \%$ & $2,75 \%$ & $15,27 \%$ \\
\hline Patronato & 1,56 & 4.346 & 82.032 & 32.296 & $0,18 \%$ & $0,48 \%$ & $39,37 \%$ \\
\hline San Diego & 2,20 & 3.977 & 46.712 & 10.230 & $0,10 \%$ & $0,15 \%$ & $21,90 \%$ \\
\hline San Pablo & 2,43 & 2.666 & 165.436 & 41.362 & $0,36 \%$ & $0,62 \%$ & $25,00 \%$ \\
\hline 10 de Julio & 2,84 & 3.159 & 76.541 & 15.130 & $0,17 \%$ & $0,23 \%$ & $19,77 \%$ \\
\hline Meiggs & 3,02 & 5.489 & 106.852 & 54.684 & $0,24 \%$ & $0,81 \%$ & $51,18 \%$ \\
\hline Vicuña Mackenna & 3,17 & 2.155 & 67.642 & 2.361 & $0,15 \%$ & $0,04 \%$ & $3,49 \%$ \\
\hline Franklin & 4,45 & 2.442 & 133.595 & 41.651 & $0,29 \%$ & $0,62 \%$ & $31,18 \%$ \\
\hline Providencia Centro & 4,63 & 2.040 & 617.004 & 70.343 & $1,36 \%$ & $1,05 \%$ & $11,40 \%$ \\
\hline Parque Arauco & 8,97 & 1.523 & 215.715 & 91.005 & $0,48 \%$ & $1,35 \%$ & $42,19 \%$ \\
\hline Vitacura Centro & 10,62 & 1.565 & 207.654 & 41.143 & $0,46 \%$ & $0,61 \%$ & $19,81 \%$ \\
\hline Rotonda Quilín & 10,99 & 3.361 & 1.757 & 0 & $0,00 \%$ & $0,00 \%$ & $0,00 \%$ \\
\hline Paradero 14 & 11,15 & 1.674 & 575.303 & 176.647 & $1,27 \%$ & $2,63 \%$ & $30,71 \%$ \\
\hline Alto Las Condes & 12,08 & 4.447 & 78.694 & 38.244 & $0,17 \%$ & $0,57 \%$ & $48,60 \%$ \\
\hline MERSAN & 13,28 & 1.818 & 16.972 & 0 & $0,04 \%$ & $0,00 \%$ & $0,00 \%$ \\
\hline Centro de Maipú & 14,38 & 1.289 & 337.705 & 78.292 & $0,74 \%$ & $1,16 \%$ & $23,18 \%$ \\
\hline Portal La Dehesa & 17,08 & 1.840 & 77.268 & 8.724 & $0,17 \%$ & $0,13 \%$ & $11,29 \%$ \\
\hline Plaza Tobalaba & 19,17 & 1.777 & 182.521 & 92.907 & $0,40 \%$ & $1,38 \%$ & $50,90 \%$ \\
\hline Centro San Bernardo & 19,28 & 2.484 & 305.038 & 127.906 & $0,67 \%$ & $1,90 \%$ & $41,93 \%$ \\
\hline Puente Alto Shopping & 20,48 & 2.686 & 411.295 & 100.572 & $0,91 \%$ & $1,50 \%$ & $24,45 \%$ \\
\hline Puente Alto Centro & 21,88 & 1.591 & 355.696 & 141.636 & $0,78 \%$ & $2,11 \%$ & $39,82 \%$ \\
\hline Plaza Sur & 23,51 & 1.184 & 48.898 & 2.733 & $0,11 \%$ & $0,04 \%$ & $5,59 \%$ \\
\hline
\end{tabular}

(*) PORCENTAJE DE VIAJES COMERCIALES DE LOS ViajeS TOTALES.

FUENTE ELABORACIÓN PROPIA SOBRE LA BASE DE EOD 2006 
En el Cuadro 4 se observa el cruce de los datos derivados de la modelación (insumos) y los datos correspondientes a la EOD 2006 para viajes por semana. El Cuadro fue dividido en cuatro tramos: en el primero se toman las áreas que se encuentran colindantes al CBD; el segundo muestra áreas pericomunales en torno a Santiago Centro; el tercero presenta áreas pericentrales, y el último tramo, zonas periféricas de la ciudad.

\section{Subcentros comerciales sobrestimados}

Al revisar las zonas en función de los viajes totales y viajes comerciales, de inmediato llama la atención la identificación de subcentros comerciales con viajes inferiores al promedio por propósito comercial (8.518 viajes a la semana). Entre estos, se descartan del análisis las zonas de Rotonda Quilín y Plaza Sur, ya que responden a malls que se inauguraron con posterioridad a la toma de datos de la EOD 2006, y se descarta la situación del Portal La Dehesa, ya que su emplazamiento responde a otros criterios (áreas de mercado con gran poder adquisitivo).

Como candidatos de datos atípicos aparecen entonces el MERsan ${ }^{12}$ y el eje de Vicuña Mackenna centro ${ }^{13}$. El primero claramente es una excepción derivada de la utilización de la densidad de superficie comercial como variable. El MERSAN efectivamente corresponde a una zona de amplia superficie comercial construida, pero su utilización con este destino no es efectiva. De hecho, en la actualidad su uso ha cambiado a bodegaje, acercándose más a los usos de corte industrial que a los comerciales.

El eje Vicuña Mackenna se presenta como una zona potencial por parte del modelo exponencial y solo es dejada como tal por la estimación de residuos del modelo potencial. Si bien posee un importante crecimiento de usos comerciales, sobre todo al alero del parque Bustamante y la avenida Vicuña Mackenna, no parece adecuado considerarla como subcentro comercial en función de las estimaciones de viajes de la misma tipología. Con todo, es una zona que se encuentra sobre el promedio en cuanto a la atracción de viajes totales (promedio de 57.488 a la semana), hecho que da luces de su potencialidad.

\section{Subcentros comerciales especializados}

El primer tramo, antes de los $3 \mathrm{~km}$ desde el СвD, alberga zonas de gran dinamismo comercial, las que se caracterizan principalmente por presentar comercio especializado, situación que les ha permitido diferenciarse como subcentros del CBD. En general estas zonas se perciben en franco retroceso en su rol de subcentralidades comerciales, ante el auge de los malls. Esta situación debiera verificarse analizando la alta densidad comercial en contraste con el total de viajes realizados en la semana. En el caso del barrio San Diego, es posible establecer dicha situación. Su proporción de viajes totales está bajo el promedio del Área Metropolitana de Santiago y su proporción de viajes comerciales se encuentra levemente sobre el promedio. Sin embargo, no es el caso de las otras tres zonas. El barrio Patronato recibe casi tantas

12 Mercado de Santiago, localizado en la comuna de Lo Espejo, cuenta con una superficie de 50 ha. Originalmente estaba destinado a la creación de un mercado; no obstante, hoy funciona como un gran centro de bodegaje.

13 Zona que se desarrolla en torno al eje Vicuńa Mackenna entre las avenidas Matta y Nuble. 
visitas como el centro comercial Alto Las Condes (considerado un shopping center regional) y la zona en torno a la avenida San Pablo recibe más de tres veces el promedio de viajes totales de Santiago.

En resumen, estas zonas aparecen como subcentralidades comerciales de peso, gracias a su especialización, situación que predispone a grandes desplazamientos para acceder a ellas. Por su pasado y por su vinculación con el CBD poseen un gran volumen de superficie comercial, lo que implica una importante potencialidad si se mantiene la estructura urbana actual de Santiago. Sin embargo, si la evolución de la estructura urbana tendiera a acentuar la policentralidad en Santiago, estas zonas podrían dejar de destacar como candidatos a subcentros.

\section{Subcentros candidatos a centros metropolitanos}

Entre los subcentros comerciales destacan dos zonas, Providencia y paradero 14 de la avenida Vicuña Mackenna, las que, a pesar de tener características diametralmente distintas, se pueden jerarquizar bajo una misma denominación: candidatos a centros metropolitanos.

La zona de Providencia tiene como particularidad ser la tercera área de la Región Metropolitana que más atrae viajes (en el contexto de las 789 áreas homogéneas). Sin embargo, en el contexto comercial aparece con una proporción de viajes marginal en comparación a los viajes totales $(11,4 \%)$. Esto se explica por el hecho de que Providencia ha alcanzado una diversificación de actividades similar a la del CBD, coexistiendo usos comerciales, de oficina, administración pública, servicios, educación, entre otros. Para esta zona la clasificación no es nueva, ya que muchas veces ha sido denominada como parte del desplazamiento del CBD desde el centro histórico, pasando por los sectores de Providencia, El Golf y actualmente Nueva Las Condes. De hecho, Ortiz y Escolano (2005) resaltan cómo el centro histórico ha ido perdiendo su imagen funcional urbana de centro comercial tradicional frente a Providencia.

El caso del paradero 14 de Vicuña Mackenna es diametralmente opuesto. Esta zona se desarrolló inicialmente en torno al mall Plaza Vespucio, y debido a su localización y a la fuerte evolución demográfica de la comuna de La Florida y Puente Alto, fue adquiriendo una importancia inusitada. Tanto es así, que alrededor de esta zona se comenzaron a localizar actividades comerciales anexas, las que posteriormente se vieron complementadas por usos de entretenimiento, educación e incluso salud. Finalmente, el desarrollo del subcentro alcanzó un grado de consolidación mayor con la localización del mall Florida Center y la densificación residencial que se ha dado en torno a este.

En términos cuantitativos, esta centralidad se encuentra inmediatamente después del centro histórico del Área Metropolitana de Santiago: posee la mayor superficie comercial de los subcentros identificados, la mayor cantidad de viajes comerciales, el tercer puesto en viajes totales y la mayor extensión territorial de los centros detectados (mayor aún que el $\mathrm{CBD}^{14}$ ).

Aunque con una densidad constructiva menor. 


\section{Candidatos a subcentros comerciales populares}

En la segunda categoría de distancia (3 a $5 \mathrm{~km}$ ) destacan dos subcentros de gran importancia en cuanto al número de viajes y alta densidad comercial. En tal sentido, el más característico corresponde al barrio Meiggs, colindante a la Estación Central de ferrocarriles, que desde los primeros análisis de datos se destacó por su altísima densidad comercial, solo superada por el CBD. Meiggs, además, se presenta como el subcentro más comercial de los subcentros analizados, apreciación basada en los datos de la EOD, que indican que sobre el $50 \%$ de los viajes que se realizan a esta zona tienen un propósito comercial (el promedio de Santiago es alrededor de 13\%). Destaca también la zona de Franklin, con casi 130.000 viajes semanales y una oferta algo más diversificada que la de Meiggs.

La importancia de estos centros radica en que se han convertido en zonas de oferta dirigida a población de más bajos ingresos, es decir, especies de centros comerciales populares. De hecho, su comparación con los malls no parece descabellada, ya que a pesar de contar con menos servicios asociados, en su conjunto y considerando un área más reducida, doblan la superficie de grandes subcentros comerciales, solo siendo superados por la del CBD y el paradero 14 de Vicuńa Mackenna.

\section{Centros comunales periféricos de alto impacto demográfico}

A partir de los $14 \mathrm{~km}$ de distancia desde el $\mathrm{CBD}^{15}$, se puede reconocer una serie de candidatos a subcentralidades comerciales que responden principalmente a dos criterios: comunas que se encontraban divididas espacialmente de la ciudad (fuera del continuo urbano), y con alto peso demográfico (entre 250.000 y 700.000 habitantes). Estos dos hechos las relevan como centralidades que se desarrollaron en función de áreas de mercado locales, evolucionando a subcentralidades metropolitanas. Esto se debe al reforzamiento de funciones centrales con el agregado de nuevas modalidades comerciales, como malls y tiendas de retail (De Mattos, 2006).

Entre las comunas con sectores candidatos a subcentros comerciales destacan, en orden de peso demográfico, Puente Alto, Maipú, San Bernardo y Quilicura. Las tres primeras presentan subcentros en sus centros históricos, los que efectivamente se han visto reforzados por la localización de malls. En el caso de Quilicura, no ha alcanzado aún el peso demográfico para la subcentralidad; no obstante, la evolución demográfica de esta comuna, la colocación de tiendas de retail en su centro y la detección por parte del modelo en el segundo umbral, la posicionan como un subcentro comercial potencial.

En función de la movilidad, se puede destacar que esta tipología de subcentro, después de la tipología de subcentros a candidatos a centro metropolitano, comprende a los que mueven mayor cantidad de viajes en Santiago (sobre 300.000 viajes semanales), siendo un tercio de estos (32\% en promedio) viajes con propósito de compras.

Es importante destacar el caso de Puente Alto, que por su gran peso demográfico (492.000 habitantes al año 2002 y más de 700.000 habitantes proyectados) genera un área de gran peso comercial al norte del centro formal, área que perfectamente podría tomarse como un solo gran subcentro lineal a lo largo de la avenida

Distancia efectiva medida por medio de análisis de redes. 
Concha y Toro, que atraviesa la comuna de norte a sur. En síntesis, esta tipología de subcentralidades comerciales en función de la jerarquía puede ser seńalada como la segunda más relevante en cuanto a movilidad y demanda.

\section{Malls como subcentros efectivos}

Cuando aparecieron los malls en Santiago, muchos urbanistas y planificadores señalaron su irrupción como el fin de la ciudad, pero el paso del tiempo se ha encargado de situarlos como subcentralidades efectivas, las que muchas veces superan el mero ámbito comercial. Es más, desde la perspectiva de la planificación, la localización de los malls ofreció una alternativa concreta al emplazamiento de los subcentros planificados por el Plan Regulador Metropolitano de Santiago, los que, a la postre, nunca se consolidaron.

Sin querer emitir un juicio al respecto, lo cierto es que, en parte importante, las subcentralidades comerciales de Santiago han nacido a partir de un mall, o bien están apoyadas por la localización de uno de ellos. De hecho, de los veintisiete malls que hoy existen en Santiago, doce se encuentran localizados en algunos de los candidatos a subcentros detectados por el modelo paramétrico. A su vez, de las veintiuna zonas detectadas como subcentros, diez de ellas cuentan con al menos un mall. En el Cuadro 5 se presentan estadísticas de los malls generalizadas en las zonas. Esto, además, permitirá caracterizar y jerarquizar en el contexto de subcentros basados en malls o eventualmente en otras categorías.

CUADRO 5 | Subcentros comerciales y estadísticas de malls

\begin{tabular}{|l|c|c|c|c|c|c|c|c|}
\hline \multicolumn{1}{|c|}{ NOMBRE } & $\begin{array}{c}\text { DISTANCIA } \\
\text { CBD }\end{array}$ & $\begin{array}{c}\text { AÑ́ } \\
\text { PRIMER } \\
\text { MALL }\end{array}$ & $\begin{array}{c}\text { AÑ́ } \\
\text { ÚLTIMO } \\
\text { MALL }\end{array}$ & $\begin{array}{c}\text { LOCALES } \\
\text { COMERC. }\end{array}$ & $\begin{array}{c}\text { AFLUENCIA } \\
\text { ANUAL }\end{array}$ & $\begin{array}{c}\text { SERVI- } \\
\text { CIOS }\end{array}$ & $\begin{array}{c}\text { EDUCA- } \\
\text { CIÓN }\end{array}$ & $\begin{array}{c}\text { ENTRETENI- } \\
\text { MIENTO }\end{array}$ \\
\hline $\begin{array}{l}\text { Paradero 14/Plaza } \\
\text { Vespucio }\end{array}$ & 11,15 & 1990 & 2003 & 481 & 58.210 .000 & 25 & 3 & 13 \\
\hline Rotonda Quilín & 10,99 & 2006 & 2006 & 52 & 3.000 .000 & 3 & 0 & 0 \\
\hline $\begin{array}{l}\text { Providencia } \\
\text { Centro }\end{array}$ & 4,63 & 1988 & 1988 & 82 & 7.000 .000 & 1 & 0 & 0 \\
\hline Parque Arauco & 8,97 & 1982 & 1982 & 287 & 27.022 .000 & 5 & 0 & 1 \\
\hline Alto Las Condes & 12,08 & 1993 & 1993 & 197 & 24.000 .000 & 3 & 0 & 2 \\
\hline Centro de Maipú & 14,38 & 1992 & 1992 & 27 & S/I & 1 & 0 & 0 \\
\hline $\begin{array}{l}\text { Centro de San } \\
\text { Bernardo }\end{array}$ & 19,28 & 1997 & 1997 & 56 & S/I & 0 & 0 & 2 \\
\hline $\begin{array}{l}\text { Puente Alto } \\
\text { Shopping }\end{array}$ & 20,48 & 1998 & 1998 & 72 & S/I & 3 & 0 & 1 \\
\hline Portal La Dehesa & 17,08 & 1992 & 2003 & 178 & 11.400 .000 & 3 & 0 & 1 \\
\hline Plaza Tobalaba & 19,17 & 1998 & 1998 & 138 & 12.500 .000 & 7 & 1 & 0 \\
\hline Plaza Sur & 23,51 & 2008 & 2008 & 150 & 15.000 .000 & 1 & 0 & 6 \\
\hline
\end{tabular}

FUENTE LABORATORIO URBANO, SOBRE LA BASE DE CÁMARA DE COMERCIO 
$\mathrm{Al}$ considerar las estadísticas, es posible profundizar en las subcategorías dentro de esta clasificación. De inmediato destaca el subcentro del paradero 14 de Vicuña Mackenna. La afluencia anual de este subcentro es solo comparable a las de los malls del sector oriente en conjunto, lo mismo que sucede con la cantidad de locales comerciales. Pero quizá lo más destacable tiene que ver con la diversificación de actividades de esta zona. Por ejemplo, la cantidad de servicios que ofrece (25) es solo equiparable a la cantidad de servicios de todo el resto de las zonas (27). Esto claramente lo sitúa en una categoría diferente, como se indicó con anterioridad.

Destacan también los malls de la zona oriente (Alto Las Condes y Parque Arauco), los que, en términos de afluencia y cantidad de recintos comerciales, no tienen competencia con el resto de los subcentros. Esto, a diferencia de los otros casos, no responde únicamente a un criterio demográfico, sino que está directamente ligado a la distribución socioeconómica de la demanda. Dicho de otra forma, al tener un poder adquisitivo mayor que el de otras zonas, el área de mercado de estos subcentros no necesita umbrales demográficos altos para su existencia, lo que justifica, entre otras cosas, su vecindad (Galetovic, Poduje \& Sanhueza, 2009). Esta es la misma razón por la cual el modelo detecta como subcentro la zona correspondiente al Portal La Dehesa, zona que -al igual que el paradero 14- posee dos centros comerciales, para un área que en contraposición tiene muy baja densidad de población, pero un enorme poder adquisitivo.

Además se evidenció de que los malls que no generan subcentros comerciales tienen muy baja diversificación de servicios en su interior y también en su entorno. De hecho, por su superficie comercial, los malls como Plaza Norte debieran aparecer detectados, lo que no sucede, debido a que se localizan en un entorno predominantemente industrial. Por las características propias de las áreas con industrias, tal localización no favorece el desarrollo de actividades complementarias a las comerciales $y$, en consecuencia, redunda en una baja densidad comercial.

\section{Conclusiones}

Densidad de superficie de uso comercial como variable para la identificación de subcentros. Se confirma la viabilidad de la utilización de la variable superficie de uso comercial como una fuente válida para la determinación de subcentralidad comercial. Esto se sustenta en la posibilidad de contar con un catastro de información universal para la ciudad de Santiago, lo que les entrega consistencia a los datos analizados. Además, el buen ajuste de dos de los modelos paramétricos ocupados permite confirmar la competencia de la variable. Es necesario mencionar que para la correcta identificación y caracterización de los candidatos a subcentros se debe complementar el análisis con datos anexos, como la movilidad, grupos socioeconómicos y otras variables que aporten en la profundización cualitativa y cuantitativa de la identificación de centralidades.

Precios de suelo como variable para la identificación de subcentros. Se rechazó la hipótesis tendiente a la utilización de esa variable para la identificación de subcentros comerciales. La aplicación del modelo demostró que se detecta como subcentros 
una gran cantidad de áreas residenciales con población de grupos socioeconómicos medios altos y altos, que no corresponden a subcentros comerciales. Este problema podría darse también en una identificación más general de subcentros de empleos.

Estructura urbana de Santiago. Por medio de la aplicación de los modelos paramétricos exponencial y potencial -en su formulación aditiva-, fue posible constatar la estructura policéntrica de Santiago, desde la perspectiva comercial. Dicha estructura tiene matices de corte monocéntrico, ya que aún se encuentra mayoritariamente dominada por el СвD. Observando la evolución de la estructura urbana de Santiago, fue posible constatar, entre los años 2001 y 2009, la disminución de la influencia del свр у la evolución de la estructura urbana hacia un modelo cada vez más policéntrico -siempre desde la perspectiva comercial-. Esto se explica por la aparición, consolidación y evolución de numerosos subcentros comerciales, en especial los que basan su poder comercial en los malls y en entornos de gran potencia demográfica (o, en su defecto, socioeconómica).

Variable independiente. Se rechazó la hipótesis tendiente al mejor ajuste del modelo por medio de la utilización de los tiempos de viaje públicos y privados en la ciudad. No obstante, se destaca el buen ajuste obtenido con el tiempo en transporte público. Esto permite suponer que ante una calibración más ajustada de la red de transporte se podría mejorar el coeficiente de determinación, con variables como la densidad de superficie comercial. En la misma línea, para el caso del transporte privado se alcanzan magros resultados, ya que la red de transporte no es consistente entre los valores de tiempo que entrega en función a la accesibilidad real. Esto se debe sobre todo a la longitud de las autopistas concesionadas. Como recomendación, se sugiere calibrar el modelo en hora peak con indicadores de saturación o nivel de servicio.

Clasificación de subcentros comerciales. Se obtuvo a una clasificación de subcentros basada en la modelación, datos de movilidad y concepciones cualitativas de las áreas estudiadas. Se plantea la división en subcentros: i) candidatos a centros metropolitanos; ii) subcentros especializados; iii) subcentros populares; iv) centros comunales de alto impacto demográfico; y v) subcentros mall. Como se pudo observar en el Cuadro 4, esta clasificación no solo implica características cualitativas, sino que tiene una impronta de jerarquía en función del tamaño y la distancia.

Malls como subcentros. Se confirma que los malls funcionan, en la mayoría de los casos, como subcentralidades comerciales, localizándose siempre en función de la demanda bajo los parámetros de umbrales demográficos y socioeconómicos correspondientes. Independientemente de su valoración subjetiva como subcentros, lo cierto es que han respondido a dicha categorización. Esto por medio de la diversificación de la actividad comercial, principalmente por la entrega de servicios complementarios, los que permiten reemplazar viajes al СвD (principalmente por parte de población periférica). Desde el punto de vista de la planificación, muchos subcentros se han transformado además en alternativas no reguladas de los subcentros propuestos por parte de instrumentos de regulación (Plan Regulador 
Intercomunal de Santiago [PRIS], 1960; Plan Regulador Metropolitano de Santiago [PRMs], 1994-1997). En tal sentido, cabe destacar que son pocos los aciertos o superposiciones entre la localización de los malls y dichas centralidades planificadas.

\section{Recomendaciones}

Los modelos paramétricos deben utilizarse considerando datos consistentes y totalizantes en función del área de estudio analizada. En tal sentido, los catastros o censos muestran claras ventajas sobre datos derivados de encuestas -muestra representativa y proyectada en el territorio-, y más aún sobre datos publicados de fuentes de información especializadas (ofertas de precio de suelo, guías comerciales, entre otros).

Para avanzar desde la identificación de candidatos a subcentros comerciales a subcentralidades comerciales formales, es necesario cumplir al menos con dos exigencias:

- Realizar una corrección de la matriz de varianzas y covarianzas por heterocedasticidad, para verificar la problemática de variables omitidas que pueden ocurrir en este tipo de modelaciones.

- Analizar de manera individual las subcentralidades comerciales de forma de validar los candidatos propuestos. Para esto se sugiere seguir el método descrito por Muñiz, Galindo y García (2005) en su artículo sobre descentralización, integración y policentrismo en Barcelona.

Ambos puntos se enmarcan en la continuación de la presente línea de investigación, que requiere ser desarrollada también en el resto de las áreas metropolitanas de Chile.

FIGURA 5 | Subcentros comerciales 2009. Caracterización

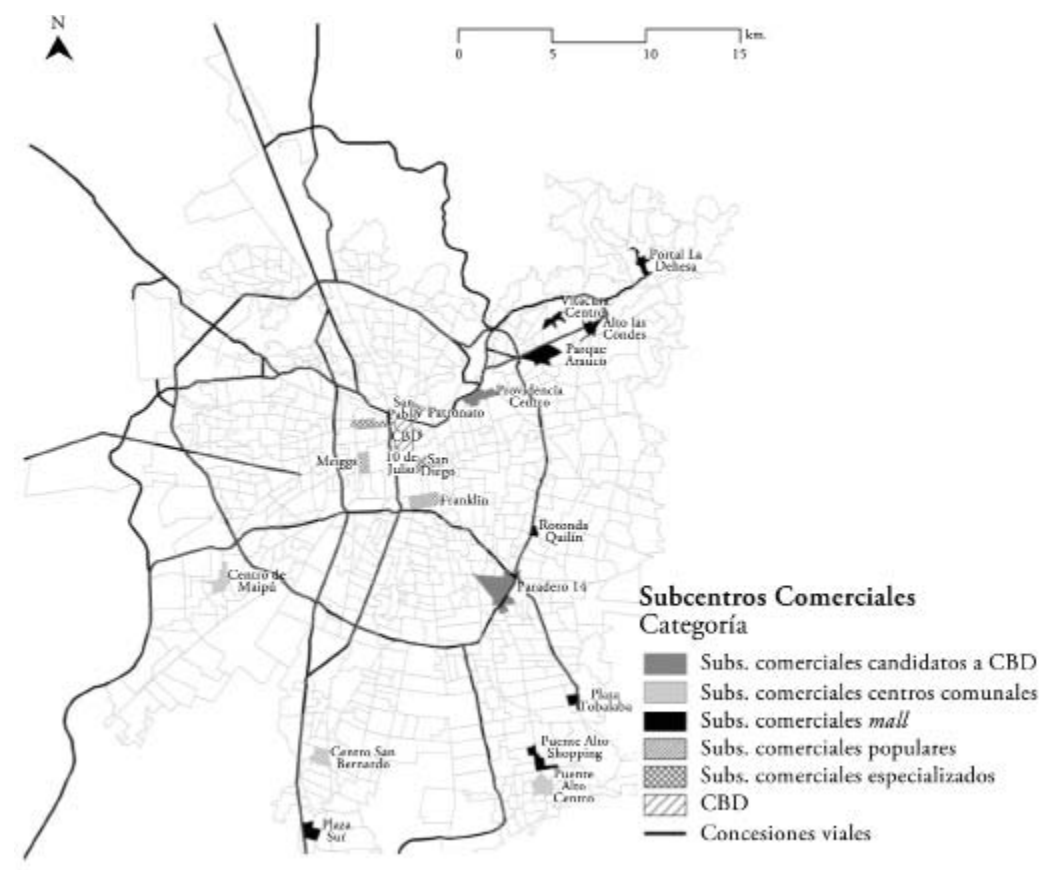

FUENTE ELABORACIÓN PROPIA 


\section{Anexos}

TABla 1 | Modelos densidad de superficie comercial 2001 en función de distancia a CBD

\begin{tabular}{|l|r|c|c|c|c|c|}
\hline COLUMNA & \multicolumn{1}{|c|}{ LINEAL } & EXPONENCIAL & ESTÁNDAR & GRAVITATORIO & LOG - LOG & POTENCIAL \\
\hline $\mathrm{R}^{2}$ & 0,147 & 0,483 & 0,316 & 0,094 & 0,351 & 0,577 \\
\hline $\mathrm{K}$ & 639,865 & 5,739 & 6,271 & 138,588 & 8,289 & $-0,178$ \\
\hline $\mathrm{a}$ & & 14,290 & & 358,727 & & $-0,044$ \\
\hline $\mathrm{b}$ & -34.853 & $-0,461$ & $-0,169$ & $-0,679$ & $-1,723$ & 1,329 \\
\hline Error est. & 479,675 & 372,91 & 1,397 & 457,09 & 1,355 & 368,1937 \\
\hline F & 135,863 & & 309,113 & & 361,285 & \\
\hline SIG & $4.47 \mathrm{E}-29$ & & $3.49 \mathrm{E}-57$ & & $9.68 \mathrm{E}-65$ & \\
\hline
\end{tabular}

TABla 2 Modelos densidad de superficie comercial 2001 en función de tiempos transporte privado a CBD

\begin{tabular}{|l|c|c|c|c|c|c|}
\hline COLUMNA & LINEAL & EXPONENCIAL & ESTÁNDAR & GRAVITATORIO & LOG-LOG & POTENCIAL \\
\hline $\mathrm{R}^{2}$ & 0,134 & 0,323 & 0,233 & 0,302 & 0,245 & 0,448 \\
\hline $\mathrm{K}$ & 686,619 & 5,745 & 6,434 & $5.818,648$ & 2,389 & $-0,176$ \\
\hline $\mathrm{a}$ & & 14,593 & & $-3.891,041$ & & $-1,09$ \\
\hline $\mathrm{b}$ & $-1140,017$ & $-10,944$ & $-5,356$ & 0,083 & -1.902 & 1,328 \\
\hline Error est. & 483,396 & 426,68 & 1,479 & 433,29 & 1,462 & 420,72 \\
\hline F & 121,639 & & 203,747 & & 216,655 & \\
\hline SIG & $2.11 \mathrm{E}-26$ & & $1.49 \mathrm{E}-40$ & & $1.09 \mathrm{E}-42$ & \\
\hline
\end{tabular}

TABla 3 | Modelos densidad de superficie comercial 2001 en función de tiempos transporte público a CBD

\begin{tabular}{|l|c|c|c|c|c|c|}
\hline COLUMNA & LINEAL & EXPONENCIAL & ESTÁNDAR & GRAVITATORIO & LOG-LOG & POTENCIAL \\
\hline $\mathrm{R}^{2}$ & 0,149 & 0,467 & 0,272 & 0,352 & 0,312 & 0,554 \\
\hline $\mathrm{K}$ & 676,493 & 5,216 & 6,379 & $5.082,156$ & 3,045 & $-2,19$ \\
\hline $\mathrm{a}$ & & 29,234 & & $-2.952,136$ & & $-2,96$ \\
\hline $\mathrm{b}$ & $-737,745$ & $-8,087$ & $-3,513$ & 0,086 & $-2,003$ & 1,138 \\
\hline Error est. & 479,201 & 378,89 & 1,441 & 417,06 & 1,359 & 378,28 \\
\hline F & 137,618 & & 250,360 & & 303,625 & \\
\hline SIG & $2.105 \mathrm{E}-29$ & & $3,79 \mathrm{E}-48$ & & $2,38 \mathrm{E}-56$ & \\
\hline
\end{tabular}


TABLA 4 Modelos densidad de superficie comercial 2009 en función de distancia a CBD

\begin{tabular}{|l|c|c|c|c|c|c|}
\hline COLUMNA & LINEAL & EXPONENCIAL & ESTÁNDAR & GRAVITATORIO & LOG-LOG & POTENCIAL \\
\hline $\mathrm{R}^{2}$ & 0,118 & 0,391 & 0,272 & 0,073 & 0,313 & 0,502 \\
\hline $\mathrm{K}$ & 670,979 & 5,953 & 6,283 & 171,454 & 8,25 & $-0,087$ \\
\hline $\mathrm{a}$ & & 11,711 & & 358,735 & & $-0,027$ \\
\hline $\mathrm{b}$ & $-35,076$ & $-0,447$ & $-0,164$ & $-0,712$ & $-1,673$ & 1,391 \\
\hline Error est. & 549,106 & 455,62 & 1,462 & 527,65 & 1,415 & 449,65 \\
\hline F & 105 & & 247,342 & & 301,397 & \\
\hline SIG & $3.24 \mathrm{E}-23$ & & $1.34 \mathrm{E}-47$ & & $6.4 \mathrm{E}-56$ & \\
\hline
\end{tabular}

TABla 5 | Modelos densidad de superficie comercial 2009 en función de tiempos transporte privado a CBD

\begin{tabular}{|l|r|c|c|c|c|c|}
\hline COLUMNA & LINEAL & EXPONENCIAL & ESTÁNDAR & GRAVITATORIO & LOG-LOG & POTENCIAL \\
\hline $\mathrm{R}^{2}$ & 0,110 & 0,259 & 0,219 & 0,184 & 0,227 & 0,397 \\
\hline $\mathrm{K}$ & 724,422 & 6,887 & 6,498 & $-101,435$ & 17,8 & $-0,09$ \\
\hline $\mathrm{a}$ & & 4,57 & & 90,023 & & $-0,692$ \\
\hline $\mathrm{b}$ & $-1163,093$ & $-10,398$ & $-5,316$ & $-1,2$ & $-1,868$ & 1,386 \\
\hline Error est. & 551,500 & 502,53 & 1,514 & 495,11 & 1,501 & 495,21 \\
\hline $\mathrm{F}$ & 97,274 & & 186,024 & & 194,258 & \\
\hline SIG & $1.03 \mathrm{E}-21$ & & $1.63 \mathrm{E}-37$ & & $6.65 \mathrm{E}-39$ & \\
\hline
\end{tabular}

TABLa 6 Modelos densidad de superficie comercial 2009 en función de tiempos transporte público a CBD

\begin{tabular}{|l|c|c|r|r|r|c|}
\hline COLUMNA & LINEAL & EXPONENCIAL & ESTÁNDAR & GRAVITATORIO & LOG-LOG & POTENCIAL \\
\hline $\mathrm{R}^{2}$ & 0,124 & 0,382 & 0,255 & 0,287 & 0,297 & 0,485 \\
\hline $\mathrm{K}$ & 717,751 & 5,430 & 6,453 & $-130,534$ & 19,422 & $-0,92$ \\
\hline $\mathrm{a}$ & & 23,852 & & 152,493 & & $-1,878$ \\
\hline $\mathrm{b}$ & $-758,661$ & $-7,841$ & $-3,494$ & $-1,205$ & $-1,988$ & 1,196 \\
\hline Error est. & 547,066 & 459,95 & 1,479 & 462,77 & 1,431 & 457,34 \\
\hline F & 111,664 & & 272,353 & & 279,352 & \\
\hline SIG & $1.69 \mathrm{E}-24$ & & $2.17 \mathrm{E}-44$ & & $1.39 \mathrm{E}-52$ & \\
\hline
\end{tabular}


TABLA 7 $\mid$ Modelos precios de suelo 2009 en función de distancia a CBD

\begin{tabular}{|l|r|c|c|c|r|c|}
\hline COLUMNA & LINEAL & EXPONENCIAL & ESTÁNDAR & GRAVITATORIO & LOG-LOG & POTENCIAL \\
\hline $\mathrm{R}^{2}$ & 0,078 & 0,078 & 0,090 & 0,083 & 0,079 & 0,797 \\
\hline $\mathrm{K}$ & 13,039 & 1,880 & 2,501 & 18,7 & 2,762 & $-4,3 \mathrm{E}-6$ \\
\hline $\mathrm{a}$ & & 2,066 & & $-4,17$ & & $-5,16 \mathrm{E}-6$ \\
\hline $\mathrm{b}$ & $-0,266$ & $-0,28$ & $-0,29$ & 0,315 & $-0,259$ & 4,7 \\
\hline Error est. & 5,113 & 5,1 & 0,516 & 5,09 & 0,519 & 5,1 \\
\hline F & 51,858 & & 60,478 & & 52,588 & \\
\hline SIG & $1.74 \mathrm{E}-12$ & & $3.14 \mathrm{E}-14$ & & $1.24 \mathrm{E}-12$ & \\
\hline
\end{tabular}

TABLA 8 Modelos precios de suelo 2009 en función de tiempo privado a CBD

\begin{tabular}{|l|c|c|c|c|c|c|}
\hline COLUMNA & LINEAL & EXPONENCIAL & ESTÁNDAR & GRAVITATORIO & LOG-LOG & POTENCIAL \\
\hline $\mathrm{R}^{2}$ & 0,033 & 0,036 & 0,28 & 0,042 & 0,028 & 0,788 \\
\hline $\mathrm{K}$ & 12,368 & 1,658 & 1,964 & 18,7 & 1,964 & $-0,00012$ \\
\hline $\mathrm{a}$ & & 2,468 & & $-4,17$ & & $-4,4 \mathrm{E}-6$ \\
\hline $\mathrm{b}$ & $-5,936$ & $-0,663$ & $-0,203$ & 0,315 & $-0,205$ & 4,72 \\
\hline Error est. & 5,236 & 5,22 & 0,533 & 5,20 & 0,532 & 5,2 \\
\hline F & 20,876 & & 17,673 & & 17,673 & \\
\hline SIG & $5.92 \mathrm{E}-6$ & & $3.014 \mathrm{E}-5$ & & $3.01 \mathrm{E}-5$ & \\
\hline
\end{tabular}

TABLA 9 | Modelos precios de suelo 2009 en función de tiempo público a CBD

\begin{tabular}{|l|r|c|r|r|r|c|}
\hline COLUMNA & LINEAL & EXPONENCIAL & ESTÁNDAR & GRAVITATORIO & LOG-LOG & POTENCIAL \\
\hline $\mathrm{R}^{2}$ & 0,028 & 0,031 & 0,024 & 0,055 & 0,036 & 0,789 \\
\hline $\mathrm{K}$ & 11,916 & 1,814 & 2,352 & 7,860 & 2,038 & $-0,000032$ \\
\hline $\mathrm{a}$ & & 2,029 & & 1,032 & & $-1.1 \mathrm{E}-6$ \\
\hline $\mathrm{b}$ & $-3,235$ & $-0,379$ & $-0,308$ & $-0,951$ & $-0,206$ & 5,12 \\
\hline Error est. & 5,250 & 5,23 & 0,534 & 5,16 & 0,531 & 5,19 \\
\hline F & 17,615 & & 15,423 & & 22,670 & \\
\hline SIG & $3.1 \mathrm{E}-5$ & & $9.5 \mathrm{E}-5$ & & $2.4 \mathrm{E}-6$ & \\
\hline
\end{tabular}

TABLA 10 | Modelos precios de suelo 2011 en función de distancia a CBD

\begin{tabular}{|l|r|c|c|c|c|c|}
\hline COLUMNA & LINEAL & EXPONENCIAL & ESTÁNDAR & GRAVITATORIO & LOG-LOG & POTENCIAL \\
\hline $\mathrm{R}^{2}$ & 0,112 & 0,115 & 0,120 & 0,117 & 0,125 & 0,775 \\
\hline $\mathrm{K}$ & 21,498 & 2,790 & 2,970 & 29,775 & 3,336 & $-0,011$ \\
\hline $\mathrm{a}$ & & 1,416 & & $-5,695$ & & $-0,0011$ \\
\hline $\mathrm{b}$ & $-0,524$ & $-0,38$ & $-0,33$ & 0,396 & $-0,326$ & 2,28 \\
\hline Error est. & 8,381 & 8,35 & 0,496 & 8,34 & 0,494 & 8,37 \\
\hline F & 88,294 & & 96,008 & & 100,061 & \\
\hline SIG & $7.74 \mathrm{E}-20$ & & $2.46 \mathrm{E}-21$ & & $4.11 \mathrm{E}-22$ & \\
\hline
\end{tabular}


TABLA 11 | Modelos precios de suelo 2011 en función de tiempo privado a CBD

\begin{tabular}{|l|r|c|r|r|r|c|}
\hline COLUMNA & LINEAL & EXPONENCIAL & ESTÁNDAR & GRAVITATORIO & LOG-LOG & POTENCIAL \\
\hline $\mathrm{R}^{2}$ & 0,056 & 0,058 & 0,052 & 0,059 & 0,052 & 0,761 \\
\hline $\mathrm{K}$ & 20,448 & 2,178 & 2,881 & 26,919 & 2,312 & $-0,022$ \\
\hline $\mathrm{a}$ & & 2,446 & & $-17,618$ & & $-0,037$ \\
\hline $\mathrm{b}$ & $-12,962$ & $-0,892$ & $-0,739$ & 0,427 & $-0,271$ & 2,16 \\
\hline Error est. & 8,638 & 8,62 & 0,515 & 8,62 & 0,514 & 8,63 \\
\hline F & 42,087 & & 38,564 & & 38,686 & \\
\hline SIG & $1.64 \mathrm{E}-10$ & & $9.06 \mathrm{E}-10$ & & $8.54 \mathrm{E}-10$ & \\
\hline
\end{tabular}

TABLA 12 | Modelos precios de suelo 2011 en función de tiempo público a CBD

\begin{tabular}{|l|r|c|c|c|c|c|}
\hline COLUMNA & LINEAL & EXPONENCIAL & ESTÁNDAR & GRAVITATORIO & LOG-LOG & POTENCIAL \\
\hline $\mathrm{R}^{2}$ & 0,059 & 0,066 & 0,066 & 0,076 & 0,085 & 0,766 \\
\hline $\mathrm{K}$ & 20,237 & 2,387 & 2,899 & 5,289 & 2,385 & $-0,000052$ \\
\hline $\mathrm{a}$ & & 2,020 & & 7,246 & & $-0,00055$ \\
\hline $\mathrm{b}$ & $-8,383$ & $-0,639$ & $-0,527$ & $-0,467$ & $-0,320$ & 3,57 \\
\hline Error est. & 8,628 & 8,58 & 0,511 & 8,52 & 0,505 & 8,55 \\
\hline F & 43,741 & & 49,4 & & 64,861 & \\
\hline SIG & $7.42 \mathrm{E}-11$ & & $4.94 \mathrm{E}-12$ & & $3.44 \mathrm{E}-15$ & \\
\hline
\end{tabular}

FUENTE ELABORACión PROPIA SObRe LA BASE DE RUIZ \& MARMOLEJO DUARTE (2008)

\section{Referencias bibliográficas}

Anas, A., Arnott, R. \& Small, K. A. (1998). Urban spatial structure. Journal of Economic Literature, 36, 1426-1463. En http://bit.ly/1xMuUMd

Borsdorf, A., Hidalgo, R. \& Sánchez, R. (2007). A new model of urban development in Latin America: The gated communities and fenced cities in Santiago de Chile and Valparaíso. Cities. The International Journal of Urban Policy and Planning, 24(5), 365378. doi: 10.1016/j.cities.2007.04.002

De Mattos, C. (2006). Modernización capitalista y transformación metropolitana en América Latina: cinco tendencias constitutivas. En A. I. Geraiges de Lemos \& M. L. Silveira, América Latina: cidade, campo e turismo (pp. 41-73). Sáo Paulo: Consejo Latinoamericano de Ciencias Sociales (CLACSO). http://bibliotecavirtual.clacso.org.ar/ ar/libros/edicion/lemos/03mattos.pdf

Dematteis, G. (1996). Suburbanización y periurbanización: ciudades anglosajonas y ciudades latinas. Presentación realizada en el ciclo "La ciudad dispersa. Suburbanización y nuevas periferias", Centre de Cultura Contemporània de Barcelona, 1996. En http:// bit.ly/1oEs6gl. Publicado en F. C. Monclús (Ed.), La ciudad dispersa. Barcelona: Centro de Cultura Contemporània de Barcelona, 1998. 
Fujita, M. \& Mori, T. (1997). Structural stability and evolution of urban systems. Regional Science and Urban Economics, 27(4-5), 399-442. doi: 10.1016/S0166-0462(97)80004-X

Galetovic, A., Poduje, I. \& Sanhueza, R. (2009). Malles en Santiago: de centros comerciales a centros urbanos. Estudios Públicos, 114 (otoño), 219-252. En http://www.cepchile.cl/ dms/archivo_4432_2576/rev114_galetovic-otros_malles.pdf

Giuliano, G. \& Small, K. A. (1991). Subcenters in the Los Angeles region. Regional Science and Urban Economics, 21(2), 163-182. doi: 10.1016/0166-0462(91)90032-I

Gordon, P. \& Richardson, H. W. (1996). Beyond polycentricity: the dispersed metropolis, Los Angeles 1970-1990. Journal of the American Planning Association, 62(3), 289-295. doi: $10.1080 / 01944369608975695$

Hidalgo, R. \& Borsdorf, A. (2009). El crecimiento urbano en Europa: conceptos, tendencias y marco comparativo para el área metropolitana de Santiago de Chile. Estudios Geográficos, 70(266), 181-203. doi:10.3989/estgeogr.0449

Horwood, E. M. \& Boyce, R. R. (1959). The свD core-frame concept. Cap. 2 en su Studies of the central business district and urban freeway development. Seattle: University of Washington Press.

Kloosterman, R. C. \& Musterd, S. (2001). The polycentric urban region: Towards a research agenda. Urban Studies, 38(4), 623-633. doi: 10.1080/00420980120035259

Krugman, P. (1999). Desarrollo, geografía y teoría económica. Barcelona: Antoni Bosch Editor.

Marmolejo, C. R. (2013). Revisiting employment density as a means to detect metropolitan sub-centres: an analysis for Barcelona and Madrid. ACE: Architecture, City and Environment, 8(23), 33-64. doi: 10.5821/ace.8.23.25.96

McDonald, J. F. (1987). The identification of urban employment subcenters. Journal of Urban Economics, 21(2), 242-258. doi: 10.1016/0094-1190(87)90017-9

McMillen, D. P. (1996). One hundred fifty years of land values in Chicago: A nonparametric approach. Journal of Urban Economics, 40(1), 100-124. doi: 10.1006/juec.1996.0025

McMillen, D. P. (2001). Non-parametric employment subcenter identification. Journal of Urban Economics, 50(3), 448-473. doi: 10.1006/juec.2001.2228

McMillen, D. P. \& McDonald, J. F. (1997). A nonparametric analysis of employment density in a polycentric city. Journal of Regional Science, 37(4), 591-612. doi: 10.1111/00224146.00071

Muñiz, I., Galindo, A. \& García, M. A. (2003). ¿Es Barcelona una ciudad policéntrica? Documento de Trabajo n. ${ }^{\circ}$ wpdea0309, Departament d'Economia Aplicada, Universitat Autonoma de Barcelona. En http://www.ecap.uab.es/repec/doc/wpdea0309.pdf

Muñiz, I., Galindo, A. \& García, M. A. (2005). Descentralización, integración y policentrismo en Barcelona. Documento de Trabajo n. ${ }^{\circ}$ wpdea0505, Departament d'Economia Aplicada, Universitat Autonoma de Barcelona. En http://www.ecap.uab.es/repec/doc/ wpdea0505.pdf

Núñez, F. \& Schovelin, R. (2002). Modelo de precio de suelo urbano en el Gran Concepción. Revista Ingeniería Industrial, 1(1), 47-58. En http://www.ici.ubiobio.cl/revista/pdf/ rev01/47-58.PDF

Ortiz, J. \& Escolano, S. (2005). Crecimiento periférico de Santiago. ¿Hacia la desconcentración funcional de la ciudad? Scripta Nova. Revista Electrónica de Geografía y Ciencias Sociales, 9(194-04). En http://www.ub.edu/geocrit/sn/sn-194-04.htm 
Parr, J. (2004). The polycentric urban region: A closer inspection. Regional Studies, 38(3), 231240. doi: $10.1080 / 003434042000211114$

Polèse, M. (2001). Cómo las ciudades producen riqueza en la nueva economía de la información: desafíos para la administración urbana en los países en desarrollo. EURE, 27(81), 5-23. http://dx.doi.org/10.4067/S0250-71612001008100001

Secretaría de Planificación de Transporte (seCtra), Chile. (2006). Encuesta de movilidad del Gran Santiago 2006. En http://www.sectra.gob.cl/datos_e_informacion_espacial/ gran_santiago/encuestas_movilidad.html

Roca, K., Marmolejo, C. R. \& Moix, M. (2008). Urban structure and polycentrism: Towards a redefinition of the sub-centre concept. Urban Studies, 46, 2841-2868. doi: $10.1177 / 0042098009346329$

Rojas Quezada, C., Muñiz Olivera, I. \& García-López, M. Á. (2009). Estructura urbana y policéntrica en el Área Metropolitana de Concepción, EURE, 35(105), 47-60. En http://www.scielo.cl/pdf/eure/v35n105/art03.pdf

Ruiz, L. M. \& Marmolejo Duarte, C. (2008). Hacia una metodología para la detección de subcentros comerciales: un análisis para Barcelona y su Área Metropolitana.ACE: Architecture, City and Environment, 3(8), 199-217. En http://revistes.upc.edu/ojs/ index.php/ACE/article/view/2464 